\title{
IMAGING WITH THZ PULSES
}

\author{
Timothy Dorney, Jon Johnson, Daniel Mittleman, Richard Baraniuk
}

Department of Electrical and Computer Engineering, Rice University, Houston, TX 77251-1892, USA

\begin{abstract}
Recently, a real-time imaging system based on terahertz (THz) time-domain spectroscopy has been demonstrated. This technique offers a range of unique imaging modalities due to the broad bandwidth, sub-picosecond duration, and phase-sensitive detection of the $\mathrm{THz}$ pulses. This paper provides a brief introduction of the state-of-the art in $\mathrm{THz}$ imaging. It also focuses on expanding the potential of this new and exciting field through two major efforts. The first concentrates on improving the experimental sensitivity of the system. We are exploring an interferometric arrangement to provide a background-free reflection imaging geometry. The second applies novel digital signal processing algorithms to extract useful information from the $\mathrm{THz}$ pulses. The possibility exists to combine spectroscopic characterization and/or identification with pixel-by-pixel imaging.
\end{abstract}

\section{INTRODUCTION}

Until the 1980 's, the use of electromagnetic waves in the far-infrared, or terahertz $(\mathrm{THz})$, region of the spectrum was limited due to the low intensity of thermal sources and the poor sensitivity of most detectors. Many of these difficulties were overcome by the introduction of $\mathrm{THz}$ time-domain spectroscopy (THz-TDS) [1-3]. This system uses femtosecond pulses of near-visible laser light to optoelectronically generate a coherent $\mathrm{THz}$ wave. The resulting electromagnetic pulse is broadband and spans from below $100 \mathrm{GHz}$ to more than $2 \mathrm{THz}$. The receiver structure requires the simultaneous arrival of a delayed femtosecond laser pulse and the generated $\mathrm{THz}$ wave. Through this arrangement, the laser pulse acts as a gating signal to control sampling. The result is a system that provides extremely bright, coherent emissions onto a gated receiver with sensitivity several orders of magnitude higher than most bolometric (thermal) counterparts.

The first real-time $\mathrm{THz}$ imaging system was introduced in 1995 [4]. In [4] and others that followed, a broad range of applications were demonstrated. The images, created from 'T-rays', included moisture analysis in a leaf, thermal analysis of a flame, and leadframe imaging of a plastic encapsulated integrated circuit, just to name a few [4-7].

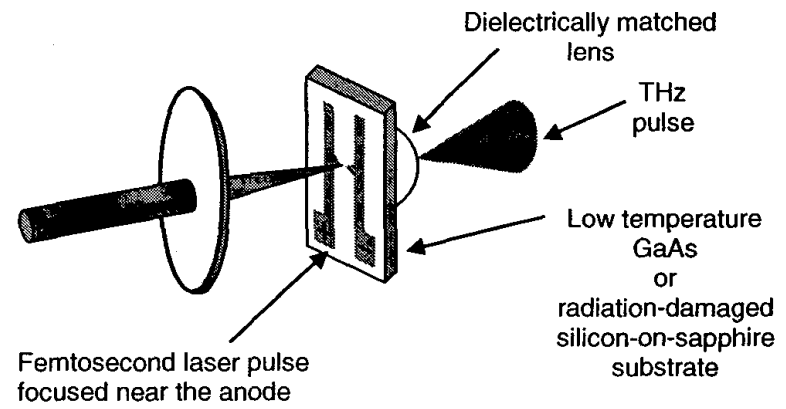

Fig. 1. The Auston switch generates $\mathrm{THz}$ pulses by firing a femtosecond laser pulse onto a DC biased antenna on a semiconductor substrate.

While a broad range of possible applications were presented, the signal processing techniques were often limited in scope. Average received power or arrival time shifts were often used to determine a false color image. This paper focuses on improving the $\mathrm{THz}$ system sensitivity through interferometry and generating spectroscopic estimations using digital signal processing.

\section{TERAHERTZ TIME-DOMAIN SPECTROSCOPY}

The heart of the $\mathrm{THz}$ system relies on two key components: the Auston switch and a visible laser capable of generating femtosecond $\left(10^{-15} \mathrm{sec}\right)$ pulse trains [1]. The Auston switch is composed of a lithographed dipole antenna patterned on a photoconductive substrate (see Fig. 1). When the dipole antennas are DC biased, a photoinduced current can be generated by the laser pulses. This current rises from zero in a time determined by the duration of each laser pulse, which is typically 0.1 picoseconds. This rapid change in current produces an electromagnetic wave in the $\mathrm{THz}$ spectral range.

The receiver optics and switch are similar to the transmitter arrangement just described. The receiver switch, however, is not DC biased. Instead, a current meter is connected across the dipole. Current will only flow when a laser pulse arrives at the switch at the same time as the coherent $\mathrm{THz}$ wave. Since the laser pulse is narrow in comparison to the time duration of the T-ray 


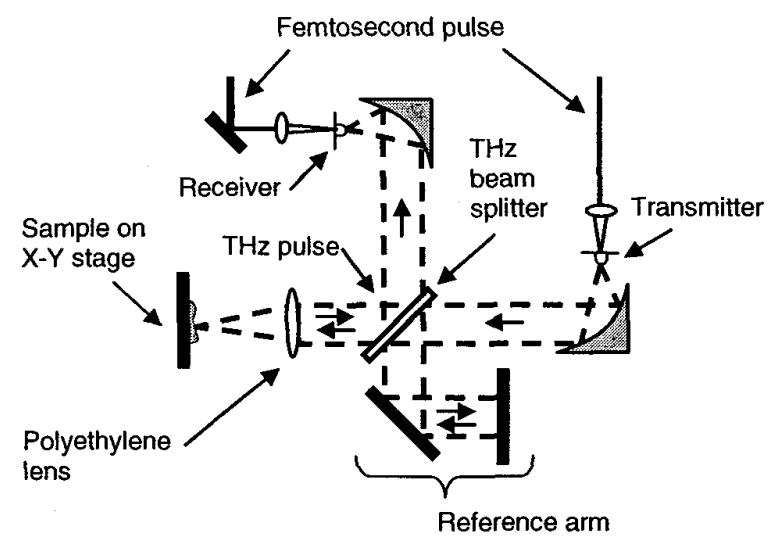

Fig. 2. Schematic of $\mathrm{THz}$ imaging with interferometry. The reference arm creates an inverted pulse that nearly cancels the main pulse at a known sample depth.

pulse, the laser acts as a gated sampling signal. The optical arrangement between the transmitter and receiver can be tailored depending on the experimental goals.

\section{TERAHERTZ INTERFEROMETRY}

$\mathrm{THz}$ imaging is an emerging technology that permits threedimensional tomographic imaging of non-metallic objects. Numerous applications have been demonstrated where $\mathrm{THz}$ imaging could be a valuable complement to existing technologies for non-invasive testing, including the detection of faults or delaminations in packaged integrated circuits and the location of air bubbles or cracks within polymer or ceramic parts $[5,6]$. In many of these applications, the feature we wish to detect is subtle, in the sense that its interaction with the single-cycle $\mathrm{THz}$ pulse imposes only a small additional distortion on the waveform. A good example is detecting delamination or disbonding between two surfaces. In many practical cases, the gap which opens between the two surfaces is narrower than the coherence length of the $\mathrm{THz}$ pulse, and the waveform is little changed as a result.

Here we report on the use of interferometry in combination with $\mathrm{THz}$ tomography, for improving the detectability of such subtle features. This idea has analogies to optical coherence tomography, in which the signal pulse, reflected off of the sample, is interfered with a reference wave to provide enhanced sensitivity [8]. The experimental layout is shown in Fig. 2. The collimated $\mathrm{THz}$ beam is directed into a Michelson interferometer, in which one arm (signal) contains a $10 \mathrm{~cm}$ polyethylene lens. Thus, the $\mathrm{THz}$ beam is focused onto the sample at normal incidence. The second arm (reference) contains a planar mirror, mounted on a translation stage for variable

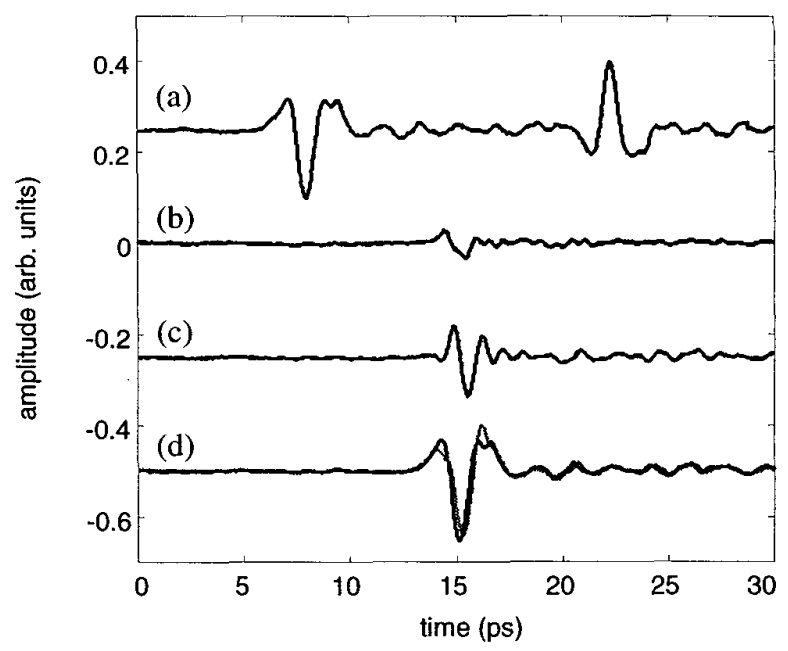

Fig. 3. $\mathrm{THz}$ waveforms with (a) a delay between the signal and reference reflections, (b) the near cancellation of the two pulses, (c) the effect on the interferometric signal caused by a piece of $45 \mu \mathrm{m}$ thick adhesive tape, and (d) the two non-interferometric signals superimposed displaying the effect of the tape.

delay. The measured signal is the coherent superposition of the electric fields from these two arms. Due to the Gouy phase shift acquired by the signal beam as it passes through a focus, it is $180^{\circ}$ out of phase from the reference beam [9]. As a result, if the sample reflects the THz pulse without distortion, then the delay of the reference arm can be adjusted so that the two pulses destructively interfere at the detector, and almost no signal is detected. Figure 3(a) depicts a waveform in which the sample and reference pulses are separated in time by a few picoseconds. Figure 3(b) shows the waveform when the two pulses are overlapped in time, producing a near cancellation of the measured signal.

This cancellation relies sensitively on the delay and distortions acquired by the signal pulse when it interacts with the sample. Any small changes in this waveform produce large fractional changes in the measured signal. This is demonstrated using waveforms reflected off of a mirror with a piece of adhesive tape stuck on the surface. The thickness of this "defect" is approximately $45 \mu \mathrm{m}$, or 1/7 of the coherence length of the pulse. The tape is composed of a transparent, low-index polymer, which has only a weak effect on the THz pulse. The waveform in Fig. 3(c) shows the effect of the tape compared to Fig. 3(b). Figure 3(d) shows the minute phase shift between waveforms using conventional (i.e., non-interferometric) imaging. The contrast is enhanced through the use of interferometry. Figure 4 shows a sample image in which this contrast enhancement is clearly evident. 


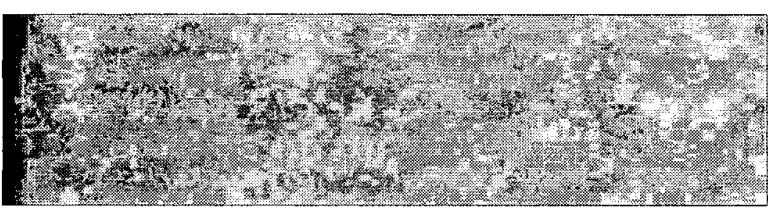

(a)

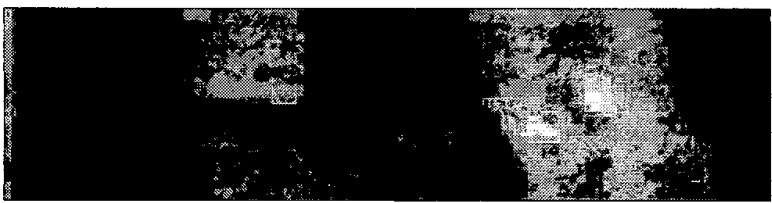

(b)

Fig. 4. Images comparing maximum peak-to-peak amplitude of (a) non-interferometric imaging, and (b) interferometric imaging. The sample consists of two $\sim 45 \mu \mathrm{m}$ thick pieces of adhesive tape on a flat metal surface. The tape strip on the right has two air bubbles.

\section{SPECTROSCOPIC ESTIMATION}

The goal in using the THz-TDS system is real-time spectroscopy and imaging. Images are formed by the collection and processing of signals in a pixel-by-pixel fashion as the sample is translated. In this work, a new technique is proposed to determine simultaneously the thickness and the complex index of refraction of an unknown material [10].

This method is a model-based approach, in which the multiple reflections arising from the time-domain FabryPerot effect are used to extract the thickness of the material as an independent measurement. A gradient search minimizes the difference between the model and the measured signals, over a range of thicknesses. Our model includes the first transmission through a material and two subsequent pulses caused by internal reflections.

The Fresnel equations describe the transmission and reflection of the $\mathrm{THz}$ wave at each interface [11]. These are based on the material's complex index of refraction in the frequency domain, $\tilde{n}(\omega)=n(\omega)-j \kappa(\omega)$, where $n(\omega)$ represents the real refractive index, $\kappa(\omega)$ is proportional to the absorption coefficient, and $\omega$ is angular frequency. The Fresnel equations at an interface between two layers are:

$$
\begin{gathered}
t_{a b}(\omega)=\frac{2 \tilde{n}_{a}(\omega)}{\tilde{n}_{a}(\omega)+\tilde{n}_{b}(\omega)}, \\
r_{a b}(\omega)=\frac{\tilde{n}_{b}(\omega)-\tilde{n}_{a}(\omega)}{\tilde{n}_{a}(\omega)+\tilde{n}_{b}(\omega)}
\end{gathered}
$$

where $t_{a b}(\omega)$ is the transmission coefficient of a wave at normal incidence from region $a$ to region $b$, and $r_{a b}(\omega)$ is the normal reflection in region $a$ at the $a-b$ interface. As the wave moves though a material of thickness $l$, its propagation is governed by:

$$
p_{m}(\omega, l)=\exp \left(\frac{-j \tilde{n}_{m}(\omega) \omega l}{c}\right) .
$$

We neglect scattering (e.g., interface roughness) in our model and consider the $\mathrm{THz}$ path both with and without a sample in place. For the free air path, we have:

$$
\begin{aligned}
& E_{\text {ref }}(\omega)=E_{\text {initial }}(\omega) p_{\text {air }}(\omega, x), \\
& \tilde{n}_{\text {air }}(\omega)=1.00027-j 0
\end{aligned}
$$

with $x$ the distance between the transmitter and receiver. This includes the small but measurable contribution of the refractive index from air at standard pressure and room temperature [12].

We examine a planar, homogenous material placed in the pathway of the $\mathrm{THz}$ radiation. Our iterative approach requires that the primary transmission and at least two multiples be present in the measured waveforms to solve for the free variables. This models the measured waveform that contains all three temporal signals:

$$
\begin{gathered}
E_{\text {complete }}(\omega)=E_{\text {initial }}(\omega) p_{\text {air }}(\omega,(x-l)) t_{01} \times \\
p_{\text {sample }}(\omega, l) t_{10} \underbrace{\left[1+\sum_{k=1}^{2}\left(r_{10}^{2} p_{\text {sample }}^{2}(\omega, l)\right)^{k}\right]}_{F P(\omega)} .
\end{gathered}
$$

We are now able to clearly discern the multiples $F P(\omega)$ described by the Fabry-Perot effect. Dividing (5) by (4), we obtain:

$$
\begin{gathered}
\hat{H}(\omega)=\frac{E_{\text {complete }}(\omega)}{\mathrm{E}_{\text {ref }}(\omega)}=\frac{4 \tilde{n}_{\text {air }}(\omega) \tilde{n}_{\text {sample }}(\omega)}{\left(\tilde{n}_{\text {air }}(\omega)+\tilde{n}_{\text {sample }}(\omega)\right)^{2}} \times \\
{\left[\exp \left(\frac{-j\left(\tilde{n}_{\text {sample }}(\omega)-\tilde{n}_{\text {air }}(\omega)\right) \omega l}{c}\right)\right] F P(\omega) .}
\end{gathered}
$$

Equation (6) provides the transfer function for our model. The complex function $\tilde{n}_{\text {sample }}(\omega)$ and $l$ are the only free variables. By minimizing this model against experimental data at each of a range of guessed thicknesses, the total error and the predicted complex index of refraction are determined. The curve created by plotting the total error for a large number of thicknesses for a sample of silicon is displayed in Fig. 5. Since the total error curve is mapped onto a decaying exponential function, the deepest local minimum determines the correct thickness. Selection of the deepest local minimum for some samples, however, is problematic. By introducing a total variation metric of the first order: $D[m]=|n[m-1]-n[m]|+|\kappa[m-1]-\kappa[m]|$,

$T V=\sum D[m]$ 


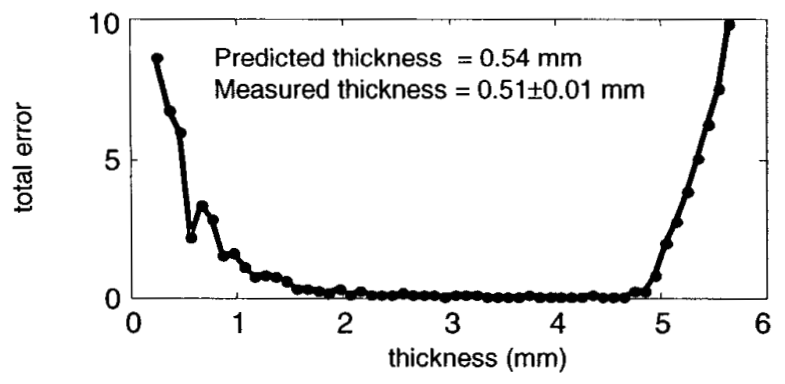

Fig. 5. The total error between the model and measured signals for a $0.51 \pm 0.01 \mathrm{~mm}$ thick sample of silicon plotted over a wide range of thicknesses.

where the complex index of refraction is $\tilde{n}(\omega)=n(\omega)-$ $j \kappa(\omega)$, we obtain the proper thickness. Total variation measures the smoothness of the refractive index at the minimum identified by the gradient descent algorithm for each thickness. We consider a wide range of possible material thicknesses. The deepest local minimum for $T V$ is more easily determined than the deepest local minimum for total error.

The deepest local minimum identified in Fig.5 is approximately $30 \mu \mathrm{m}$ from the measured value. The predicted thickness error is 10 times less than the coherence length of the terahertz pulse. The complex index of refraction at the predicted thickness is compared to literature data in Fig. 6 [13]. Both the real and imaginary predicted refractive indices are independent of frequency as expected.

\section{SUMMARY}

We have briefly explained the opto-electronic mechanisms that allow $\mathrm{THz}$ emission and detection. By introducing an interferometric arrangement, the sensitivity of the system is improved. We demonstrate qualitative assessment with an image of tape that has a thickness $1 / 7$ of the coherence length of the terahertz pulse. A non-interferometric arrangement barely resolves the tape, whereas the interferometric method clearly shows the tape and trapped air bubbles. Finally, we introduced a spectroscopic imaging method that simultaneously and independently determines the complex index of refraction and thickness of a sample.

We wish to acknowledge the support of the Army Research Office, Environmental Protection Agency, and the National Science Foundation. Further information is available at www.dsp.rice.edu/ mit.

E-mail: \{mit, johnsojl, daniel, richb\} @rice.edu

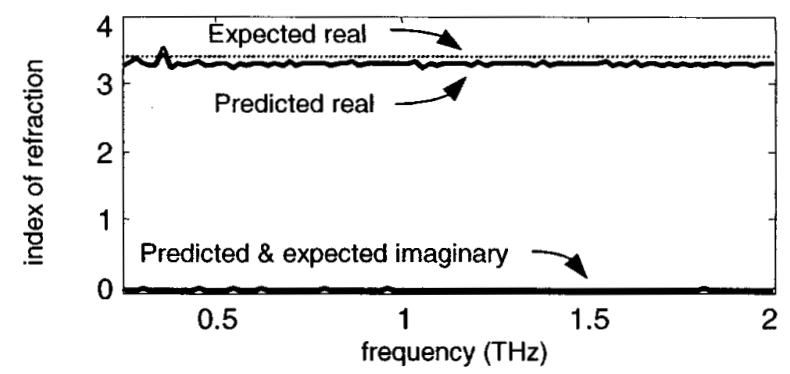

Fig. 6. The real and imaginary index of refraction for the thickness identified in Fig. 5. Literature data puts the complex index of refraction of silicon at 3.418-j0 [13].

\section{REFERENCES}

[1] P. Smith, D. Auston, and M. Nuss, "Subpicosecond Photoconducting Dipole Antennas," IEEE J. Quant. Elec., 24(2), 255-260 (1988).

[2] M. van Exter and D. Grischkowsky, "Characterization of an Optoelectronic Terahertz Beam System," IEEE Trans. Micro. Theo. Tech., 38, 1684-1691 (1990).

[3] M. Nuss and J. Orenstein, "Terahertz Time-Domain Spectroscopy (THz-TDS)," in Millimeter and Submillimeter Wave Spectroscopy of Solids, ed. G. Grüener, (Heidelberg, Germany: Springer-Verlag, 1998), and references therein.

[4] B. Hu and M. Nuss, "Imaging with terahertz waves," Opt. Lett., 20(16), 1716-1718 (1995).

[5] D. Mittleman, R. Jacobsen, and M. Nuss, "T-ray imaging," IEEE J. Sel. Top. Quant. Elect., 2(3), 679-692 (1996).

[6] D. Mittleman, S. Hunsche, L. Boivin, and M. Nuss, "T-ray tomography," Opt. Lett., 22(12), 904-906 (1997).

[7] D. Mittleman, M. Gupta, R. Neelamani, R. Baraniuk, J. Rudd, and M. Koch, "Recent advances in terahertz imaging," Appl. Phys. B, 68, 1085-1094 (1999).

[8] D. Huang, E. Swanson, C. Lin, J. Schuman, W. Stinson, W. Chang, M. Hee, T. Flotte, K. Gregory, C. Puliafito, and J. Fujimoto, "Optical coherence tomography," Science, 254, 11781181 (1991).

[9] A. Ruffin, J. Rudd, J. Whitaker, S. Feng, and H. Winful, "Direct observation of the Gouy phase shift with single-cycle terahertz pulses," Phys. Rev. Lett., 83(17), 3410-3413 (1999).

[10] L. Duvillaret, F. Garet, and J. Coutaz, "Highly Precise Determination of Both Optical Constants and Sample Thickness in Teraherz Time-Domain Spectroscopy," Appl. Opt., 38, 409415 (1999).

[11] E. Hecht, Optics, $2^{\text {nd }}$ ed. (Reading, Massachusetts. Addison-Wesley, 1987).

[12] P.E. Ciddar, "Refractive index of air: new equations for the visible and near infrared," Appl. Opt., 35, 1566-1573 (1996).

[13] D. Grischkowsky, S. Keiding, M. van Exter, and C. Fattinger, "Far-infrared time-domain spectroscopy with terahertz beams of dielectrics and semiconductors," J. Opt. Soc. Am. B, 7, 2006-2015 (1990). 